\title{
La solución de fachada convencional del periodo desarrollista en el caso de Gipuzkoa: declive (y final) de un sistema constructivo
}

\author{
The conventional facade solution during the developmental period in \\ Gipuzkoa: decline (and end) of a construction system
}

\author{
I. Lizundia-Uranga ${ }^{(*)}$
}

\section{RESUMEN}

El artículo analiza el proceso constructivo, la evolución y el inquietante futuro del cerramiento de fachada de los edificios de vivienda colectiva construidos durante el periodo desarrollista en Gipuzkoa. Se han inspeccionado 128 conjuntos residenciales y se ha entrevistado a 17 personajes que intervinieron directamente en la construcción de muchos de ellos. Tras analizar los materiales y elementos que constituyen la solución de fachada convencional utilizada en Gipuzkoa y en gran parte del conjunto del estado español durante aquellos años y cuyo uso se ha prolongado hasta nuestros días, se estudian los efectos y las causas de sus principales lesiones y carencias. Su compleja y costosa adecuación a las exigencias actuales va a requerir de un importante esfuerzo por parte del conjunto de la sociedad y plantea la cuestión de si estamos ante un sistema constructivo cuyo ciclo de vida debe darse por concluido de una vez por todas.

Palabras clave: Fachada convencional; desarrollismo; Gipuzkoa; rehabilitación.

\section{ABSTRACT}

The article analyses the construction process, the evolution and the disturbing future of the facade enclosure of the collective housing constructions built during the developmental period in the province of Gipuzkoa. 128 residential complexes were checked, and 17 people who directly took part in the construction of many of these buildings were interviewed. After examining the material and elements that make up the conventional facade solution used in Gipuzkoa and in much of Spain not only during those years but also up to the present time, the effects and causes of its main damages and flaws were studied. Its complex and costly adaptation to current demands is going to require a strong effort from society at large, and this raises the question of whether the life cycle of this construction system should be terminated once and for all.

Keywords: Conventional facade; developmentalism; Gipuzkoa; rehabilitation.

(*) ETSA - Euskal Herriko Unibertsitatea / Universidad del País Vasco. San Sebastián (España). Persona de contacto/Corresponding author: inigo.lizundia@ehu.eus (I. Lizundia-Uranga)

Cómo citar este artículo/Citation: Lizundia-Uranga, I. (2015). La solución de fachada convencional del periodo desarrollista en el caso de Gipuzkoa: declive (y final) de un sistema constructivo. Informes de la Construcción, 67(538): e079, doi: http://dx.doi.org/10.3989/ic.13.167.

Licencia/License: Salvo indicación contraria, todos los contenidos de la edición electrónica de Informes de la Construcción se distribuyen bajo una licencia de uso y distribución Creative Commons Reconocimiento no Comercial 3.o. España (cc-by-nc). 


\section{INTRODUCCIÓN}

Durante el conocido como periodo desarrollista, periodo que puede acotarse entre finales de los cincuenta y mediados de los setenta del siglo pasado, Gipuzkoa experimentará el mayor crecimiento demográfico de su historia pasando de 478.337 hab. en 1960 a 682.517 hab. en 1975 (1). Las principales causas serán la elevada tasa de crecimiento natural y la inmigración. El espectacular aumento de la demanda de vivienda se traducirá en un auténtico boom constructivo y provocará una radical transformación urbanística y social de los principales municipios de este territorio, condicionando de forma determinante su carácter y configuración futura en todos los aspectos.

Un boom que, paradójicamente, tiene todos los visos de repetirse aunque, en este caso, debido a la necesidad de rehabilitar gran parte de los edificios construidos durante esos años (2). Las múltiples lesiones y carencias que presentan algunos de sus elementos constructivos, principalmente los situados en la envolvente del edificio y muy especialmente en la fachada, están convirtiendo a estos edificios en los auténticos protagonistas de la rehabilitación en nuestros días. Una circunstancia altamente preocupante que exige poner sobre la mesa la cuestión de si estos edificios pueden haber alcanzado una edad crítica y si se ha podido producir el agotamiento último de alguno de sus elementos constructivos.

Todo ello en un contexto en el que la legislación vigente en el País Vasco obliga a que todos los edificios de uso residencial de más de cincuenta años tengan que proceder, ineludiblemente, a subsanar las deficiencias reflejadas en el correspondiente informe de la inspección técnica (ITE) a la que tendrán que ser sometidos. Unos trabajos de reparación que deberán ir, además, estrechamente vinculados a otros encaminados a dar respuesta a las exigencias impuestas por la Ley 8/2013 como resultado de la transposición a la legislación estatal de la Directiva europea 2010/31/UE relativa a la eficiencia energética de los edificios.

En el presente artículo se analiza la construcción, la evolución y el previsible futuro de la envolvente de fachada de los edificios de vivienda colectiva construidos durante el periodo desarrollista en el Territorio Histórico de Gipuzkoa.

\section{METODOLOGÍA}

Las principales fuentes de información utilizadas en la investigación han sido:

- El análisis de 128 conjuntos residenciales (cr), que incluyen 484 edificios (e) con un total de 13.433 viviendas (v) y que se sitúan en seis de los municipios que más crecieron desde el punto de vista demográfico: Donostia-San Sebastián ( 15 cr, 58 e, 2.691 v), Rentería (10 cr, 126 e, 4.953 v), Irún (13 cr, 76 e, 1.424 v), Hernani ( 8 cr, 65 e, 1.254 v), Arrasate-Mondragón ( 8 cr, 49 e, 1.383 v) y Zarautz ( 74 cr, 110 e, $1.728 \mathrm{v}$ ). Se han estudiado los proyectos originales y se ha realizado una inspección visual de cada uno de ellos.

Tres han sido los factores considerados a la hora de seleccionar los diferentes conjuntos residenciales: el número de viviendas, con el objeto de aumentar su valor estadístico; su pertenencia a épocas diversas dentro del periodo ana- lizado; y, por último, su diferente y variada localización dentro del municipio. No se ha valorado su mayor o menor calidad arquitectónica ya que el principal objetivo ha sido indagar en los rasgos constructivos fundamentales de ese gran número de edificios, la mayor parte de ellos anónimos, que conforman y definen el carácter de nuestros municipios.

- El testimonio inédito de diecisiete personajes (arquitectos, aparejadores, promotores, constructores, fabricantes de materiales y políticos, cuyo listado se recoge en el punto final Agradecimientos) que intervinieron directamente en el proceso constructivo de una buena parte de aquellos edificios. Las entrevistas realizadas han quedado recogidas en varias decenas de horas de grabación.

- La consulta de la colección completa de la revista Informes de la Construcción publicada por el Instituto Eduardo Torroja durante el periodo analizado (propiedad del arquitecto Francisco Bernabé).

\section{LA FACHADA CONVENCIONAL}

La evolución que sufrirá la fachada a lo largo del periodo analizado afectará principalmente a los revestimientos de acabado de su cara exterior. Su tipología (composición, orden, dimensión y función de sus diferentes capas) apenas sufrirá cambios.

El escaso desarrollo tecnológico del país, la falta de investigación e información sobre nuevos productos, una normativa limitada y una mano de obra acomodada a la utilización de materiales y sistemas constructivos simples, bien conocidos y avalados por la experiencia, harán que los cambios que se vayan introduciendo sean muy pausados y progresivos.

Para finales de los cincuenta, el muro de fachada habrá conseguido desembarazarse de toda misión estructural y su función se limitará al de mero cerramiento. Entre los 128 conjuntos residenciales analizados sólo se ha encontrado uno en el que el cierre exterior colabora en el sistema estructural del edificio. Así, la solución de fachada que prevalecerá, no solo en Gipuzkoa sino en gran parte del conjunto del estado, durante todo el periodo desarrollista será la formada por una doble hoja de fábrica de ladrillo, apoyada total o parcialmente sobre el borde perimetral de cada forjado y cámara de aire intercalada (Figura 1). Una solución conocida hoy como fachada convencional y que, con la única novedad consistente en la introducción de un material aislante en la cámara de aire, se sigue utilizando habitualmente en la mayoría de edificios de nueva construcción.

\subsection{La constitución de las hojas de fachada}

La información aportada en las memorias de los proyectos originales es mínima, siendo en el presupuesto y en los esquemáticos detalles constructivos donde las especificaciones sobre los materiales y los sistemas empleados comienzan a estar algo más definidas.

\subsubsection{La hoja exterior}

En el $75 \%$ de los conjuntos analizados, la hoja exterior se resuelve con una fábrica de ladrillo hueco doble revestida exteriormente. De éstos, en un 20 \% la colocación se realiza a media asta y en un $31 \%$ a tabicón, no habiendo sido posible extraer información alguna en los demás casos. En el 

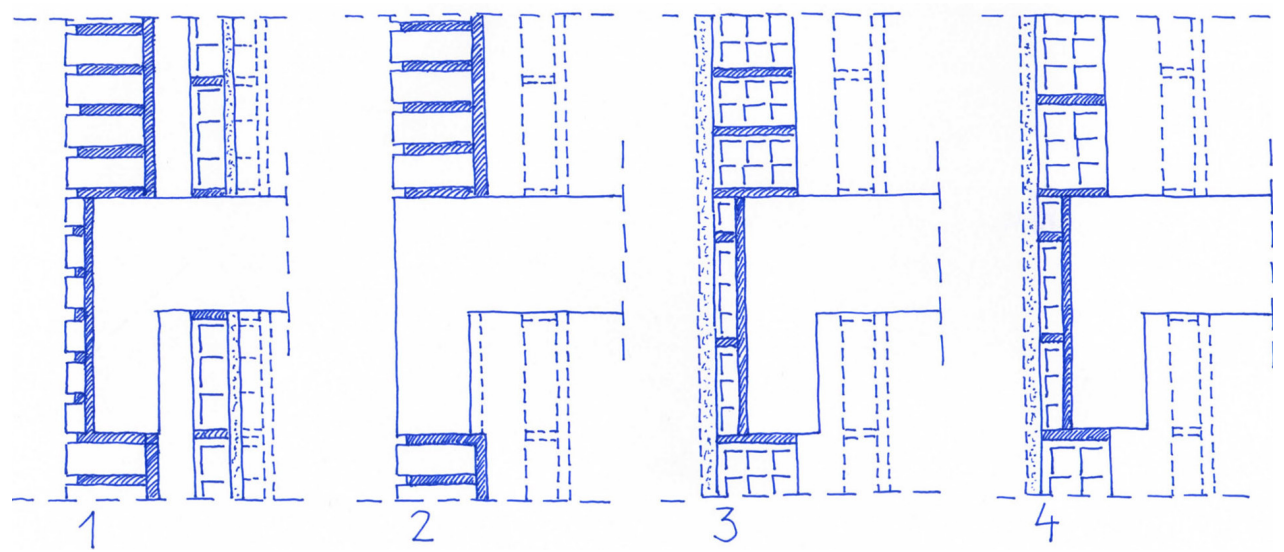

Figura 1. Cuatro variantes de la solución de fachada convencional típica de la época:

1) Hoja exterior de fábrica de ladrillo caravista con la cara interior raseada, frente estructural revestido, cámara de aire y tabique (o tabicón) interior enlucido. 2) Similar a la anterior con el frente estructural visto. 3) Hoja exterior de fábrica de ladrillo hueco doble colocado a media asta y revestida exteriormente. 4) Similar a la anterior con la fábrica exterior dispuesta a tabicón.

25 \% restante, la fábrica es de ladrillo caravista colocado a media asta.

La mayor parte del ladrillo ordinario provendrá de una serie de fábricas situadas en diferentes localidades de la provincia de Gipuzkoa. En unos primeros años en los que la única aproximación prescriptiva será la aportada por el Pliego de la Dirección General de Arquitectura de 1960 (3), las dimensiones de las piezas variarán según el fabricante. Como ejemplo, el ladrillo doble de seis huecos fabricado por una de las principales tejerías de Gipuzkoa (Tejas y ladrillos del Oria S.A. de Andoain) medirá $25 \times 12 \times 9 \mathrm{~cm}$, el ladrillo sencillo de cuatro huecos $30 \times 15 \times 4,5 \mathrm{~cm}$ y la rasilla de tres huecos $25 \times 12 \times 3 \mathrm{~cm}$.

La primera hilada apoyará sobre el borde de cada forjado y, normalmente, volará alrededor de un tercio de su anchura respecto al frente estructural. Esta disposición permitirá que los pilares, vigas y cantos de forjado puedan ser revestidos mediante piezas de menor espesor igualando y dando continuidad al plano de fachada. En las fachadas de ladrillo caravista esta pieza se obtendrá del corte parcial de un ladrillo mientras que en el resto de casos el forrado se realizará mediante ladrillos de rasilla.

La práctica totalidad de las fachadas resueltas con ladrillo caravista protegerán su cara interior, la que mira a la cámara de aire, con un enfoscado de mortero de cemento para evitar la filtración de humedad hacia el interior (variantes 1 y 2 de la Figura 1). En algunos proyectos, se especifica que el mortero será hidrófugo y en tres de los casos se prescribe, además, una imprimación asfáltica sobre su superficie. Los arquitectos entrevistados coinciden en que era la más sencilla y efectiva solución para evitar la entrada de agua al interior de la cámara a través de las fábricas de ladrillo caravista, desprovistas de cualquier revestimiento impermeable adicional por el exterior.

\subsubsection{La cámara de aire}

En los ocho casos en los que su dimensión aparece definida en proyecto, la anchura prescrita varía entre 5 y $11 \mathrm{~cm}$. Resulta sorprendente, por lo tanto, que en los cuatro casos en los que se ha tenido acceso visual a la misma (Figura 2), la anchura haya superado siempre estas dimensiones llegando incluso, en uno de los casos, hasta los $20 \mathrm{~cm}$.

De los 128 conjuntos analizados, solo dos incluyen un material aislante entre las dos hojas que conforman la fachada. Se trata de dos edificios de la última fase del periodo, proyectados en 1971 y 1974 respectivamente, para cuyas cámaras se prescriben sendas placas de poliestireno expandido de $40 \mathrm{~mm}$ de espesor, sin que en sus respectivos proyectos se aclaren los motivos de su inserción. El sorprendente ar-

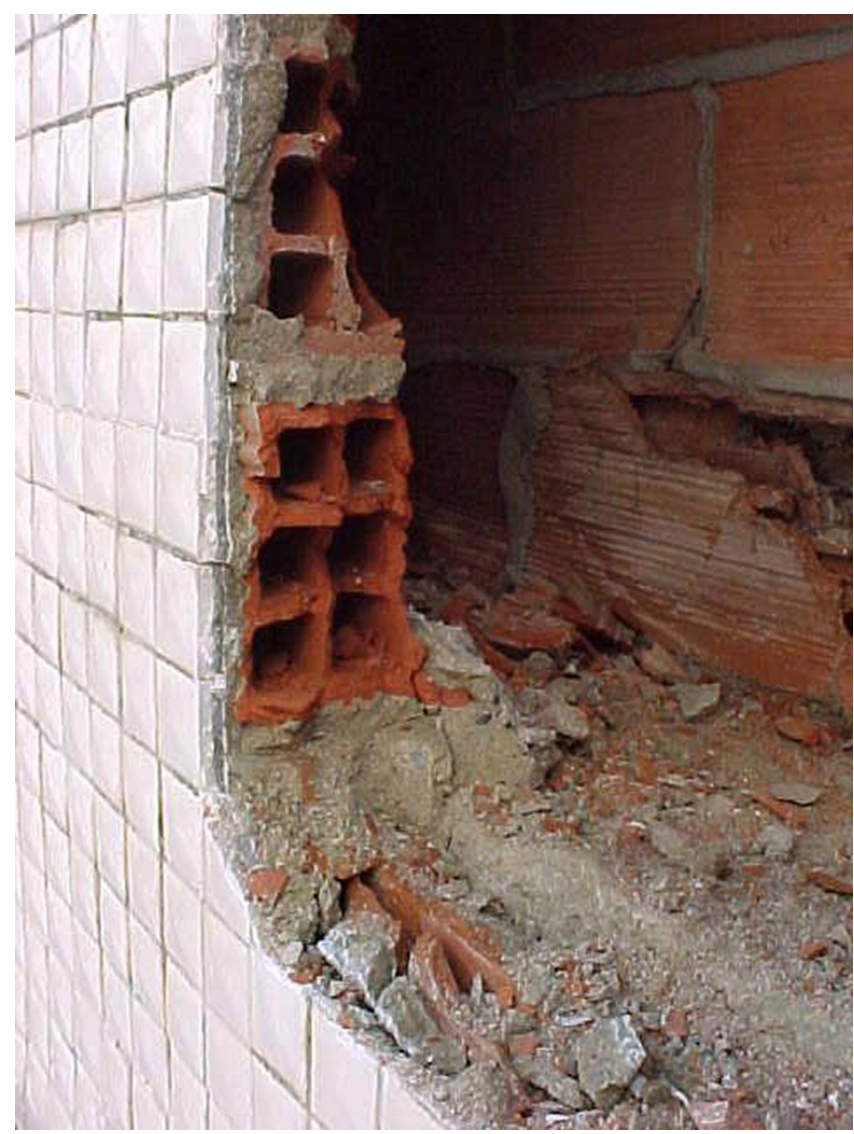

Figura 2. Cámara de aire de $13 \mathrm{~cm}$ de anchura en la fachada del bloque de 16 viviendas en Nafarroa, 39 de Zarautz, proyectado en 1970 por Roberto Martínez Anido. 
gumento esgrimido por el autor del primero de ellos, el arquitecto Armando Roca (4), para comenzar a utilizarlos no obedecerá a cuestiones térmicas ni acústicas sino puramente constructivas. Cuenta el arquitecto que, durante las visitas de obra, tanto él como el aparejador observaban con preocupación la cantidad de rebabas de mortero que caían en las cámaras de aire al levantar la hoja interior, cuya posterior eliminación resultaba muy complicada. Para evitarlo y solo para ello, decidieron comenzar a colmatar las cámaras con placas aislantes que se fijaban a la hoja exterior.

Aunque ya existe constancia de una fachada de doble hoja con aislamiento incorporado construida en Madrid allá por 1927 (5) y a pesar de que la comercialización de los primeros productos aislantes destinados a la construcción en el estado español se remonta a los años cuarenta, llegando a convertirse en algunos de los productos más publicitados en revistas técnicas como Informes de la Construcción del Instituto Eduardo Torroja, su presencia en la arquitectura residencial de Gipuzkoa será testimonial. Según todos los arquitectos entrevistados, los aspectos térmicos y acústicos no preocupaban por aquellos años.

Tampoco las normativas eran muy exigentes. Exceptuando algunos comentarios genéricos incluidos en las Normas Higiénicas de 1944 o las Ordenanzas para Viviendas de Renta Limitada de 1955, la normativa térmica vigente a principios de los setenta se reducirá a un simple artículo de las Ordenanzas Provisionales de Viviendas de Protección Oficial de 1969 en el que se limitará la conductibilidad de los elementos de la envolvente sin que, en ningún caso, se lleguen a definir las posibles soluciones constructivas para justificar su cumplimiento. No será hasta 1973, con la llegada de la crisis energética, cuando se empiece a plantear de forma más o menos seria la cuestión del ahorro energético. Un hecho que llevará a la aprobación en 1975 de un primer Decreto sobre medidas tendentes a reducir el consumo de energía (6) y que será la antesala de la primera norma térmica de obligado cumplimiento, la NBE-CT-79.

La normativa acústica, por su parte, se reducirá a unas sencillas normas de 1961 y a unas propuestas, algo más elaboradas, planteadas por el Jefe del Departamento de Construcciones del INV, José Fonseca, en el marco del Seminario de Viviendas organizado por el Ministerio de la Vivienda y celebrado en Madrid en junio de 1964 (7). Una vez más, será el Pliego de la DGA de 1960 el documento que más información aporte sobre las características y forma de empleo de los materiales aislantes diferenciando, incluso, los aislamientos acústicos de los térmicos.

\subsubsection{La hoja interior}

En el $86 \%$ de los casos en los que queda definido en proyecto, se resuelve mediante un tabique de fábrica de ladrillo hueco sencillo. Con el paso de los años y sobre todo en los edificios con mayor calidad constructiva, comienza a utilizarse el ladrillo hueco doble colocado, en todos los casos, a tabicón. El acabado interior se realizará mediante un enlucido de yeso revestido con una pintura o un papel pintado.

\subsection{El acabado exterior}

\subsubsection{Los revestimientos continuos}

Continuando con la tipología predominante en la década precedente, constituirán la solución más utilizada durante los primeros años sesenta. La práctica ausencia de fabricantes de otro tipo de materiales de acabado en el entorno próximo y una red comercial exterior todavía por desarrollar serán las causantes de que los revestimientos continuos se impongan en esos primeros años. Su utilización como solución única para toda la fachada se ha podido constatar en el $33 \%$ de los conjuntos analizados mientras que en otros muchos casos aparece combinada con otros materiales.

Para el acabado final se utilizarán pinturas de diferentes tipos y marcas comerciales y revestimientos compuestos por gránulos de mármol aglomerados con resinas como el Granulite, aplicados todos ellos sobre un enfoscado previo de mortero de cemento.

\subsubsection{Los revestimientos adheridos}

Aparecen en el $42 \%$ de los casos analizados. Predominarán los revestimientos cerámicos compuestos por baldosas y plaquetas de gres de diferentes tamaños y formatos, las plaquetas de ladrillo o ladrilletas que imitarán al ladrillo caravista y los mosaicos vítreos que, a pesar de su alto grado de vitrificación, serán en su gran mayoría de gres.

Aunque es posible encontrarlos en algunos edificios tempranos, su uso se impondrá a partir de mediados de la década de los sesenta cuando las empresas fabricantes instaladas en regiones de fuerte tradición cerámica, como el Levante o Castilla, consoliden sus redes comerciales en el País Vasco. Otros materiales que, aunque en menor medida, también se fijarán a la fachada serán las placas de piedra natural (ancladas) y piedra artificial, las baldosas hidráulicas de cemento (utilizadas por primera vez por los arquitectos Juan Manuel Encío y Luis Peña Ganchegui) y las baldosas de canto rodado.

\subsubsection{El ladrillo caravista}

Se introduce en Gipuzkoa a mediados de los años sesenta aunque será en la década de los setenta cuando adquiera verdadero protagonismo. El porcentaje de edificios en los que se emplea como solución única alcanza el $17 \%$ del total de casos analizados. En ocasiones, aparece combinado con otro tipo revestimientos o con el hormigón picado o abujardado de los frentes estructurales vistos de fachada. El hecho de poder resolver el soporte y el acabado de la hoja exterior mediante un único producto supondrá una auténtica novedad tanto en lo relativo al proceso constructivo como a las posibilidades de diseño y composición, adquiriendo gran aceptación por parte de proyectistas y constructores pese a la inicial reticencia de algunos arquitectos debido a la falta de mano de obra especializada.

A diferencia del ladrillo común, las tejerías locales no elaboraban este tipo de ladrillo y era necesario traerlo de zonas como Tudela, Albacete, Palencia, Jaén o Madrid. Hacia 1970 y ante el fuerte incremento de la demanda, la ya citada Tejas y ladrillos del Oria S.A. de Andoain decide comenzar a fabricarlo aunque la ausencia de una arcilla de calidad en el entorno próximo impedirá la elaboración de un ladrillo de acabado fino, presentando una terminación basta y desigual. La empresa lo comercializará bajo la denominación de ladrillo rústico, llegando a tener una buena aceptación.

En ninguno de los proyectos se hace mención alguna a las características exigibles al ladrillo, al mortero, al aparejo, a la junta o al control de puesta en obra. El espesor de la fábri- 
ca variará entre 10 y $12 \mathrm{~cm}$ empleándose, en todos los casos, el aparejo a sogas, es decir, aquel en el que el ladrillo se coloca a soga (o a media asta) quedando el grosor condicionado por la dimensión del tizón. El importante rehundido de las juntas de las llagas y tendeles se convertirá en uno de los rasgos característicos de las fábricas de ladrillo caravista de la época.

\subsection{El perímetro del hueco de fachada}

Una de las soluciones más recurrentes a la hora de resolver el dintel exterior será la de aprovechar el importante descuelgue de la viga situada en el borde del forjado. Bastará con dimensionar su canto de tal modo que, independientemente de las demandas estructurales, se ajuste a las dimensiones exigidas por el hueco de ventana. En la parte interior, el tramo superior del hueco se completará con la caja de persiana o, en su ausencia, mediante una fábrica de ladrillo hueco sencillo apoyada directamente sobre el grueso perfil superior de la carpintería de madera de pino de la ventana, material habitual durante todo el periodo hasta la irrupción, a principios de los setenta, de las primeras carpinterías de aluminio.

Otras soluciones menos habituales para resolver el dintel serán las piezas prefabricadas cerámicas o de hormigón, los dinteles de hormigón armado realizados in situ y revestidos exteriormente con ladrillos caravista colocados a sardinel y, excepcionalmente, los arcos de correa de ladrillo y los perfiles de acero.

En cuanto a los alféizares, los más utilizados serán los de hormigón prefabricado y piedra artificial. Se apoyarán sobre las dos hojas de fábrica de ladrillo y atravesarán todo el espesor de la fachada quedando su testero interior enrasado con el tabique. La carpintería de ventana montará directamente sobre ella sin la ayuda de ningún tipo de premarco.

La sección constructiva típica del hueco de fachada de un edificio de la década de los sesenta se recoge en la Figura 3.

\subsection{Los vuelos de fachada}

La mayoría de las estructuras de los balcones, en ocasiones de considerable vuelo, se plantearán como una prolongación del forjado unidireccional, de entre 16 y $26 \mathrm{~cm}$ de espesor, con las viguetas dispuestas en sentido perpendicular a la línea de fachada. El perímetro se rematará con una viga de borde armada de forma desigual que presentará, en su parte inferior y en toda su longitud, un rebaje a modo de goterón.

Los balcones y aleros construidos mediante losas macizas de hormigón armado serán, en general, de menor sección. Entre los ejemplos analizados, se han encontrado losas con espesores de 10,12, 13, 15 y $18 \mathrm{~cm}$. La cara superior sometida a tracción contará con una armadura principal formada por una serie de barras dispuestas en sentido transversal que se prolongarán sobre el forjado en una longitud similar a la del vuelo. El armado superior se completará con unas varillas de reparto paralelas a la fachada, mientras que el armado de la cara inferior se producirá sólo en contadas ocasiones.

Dos de las características más reseñables de los balcones de la época serán la ausencia generalizada de una membrana impermeabilizante bajo el acabado (sólo se prescribe en el $16 \%$ de

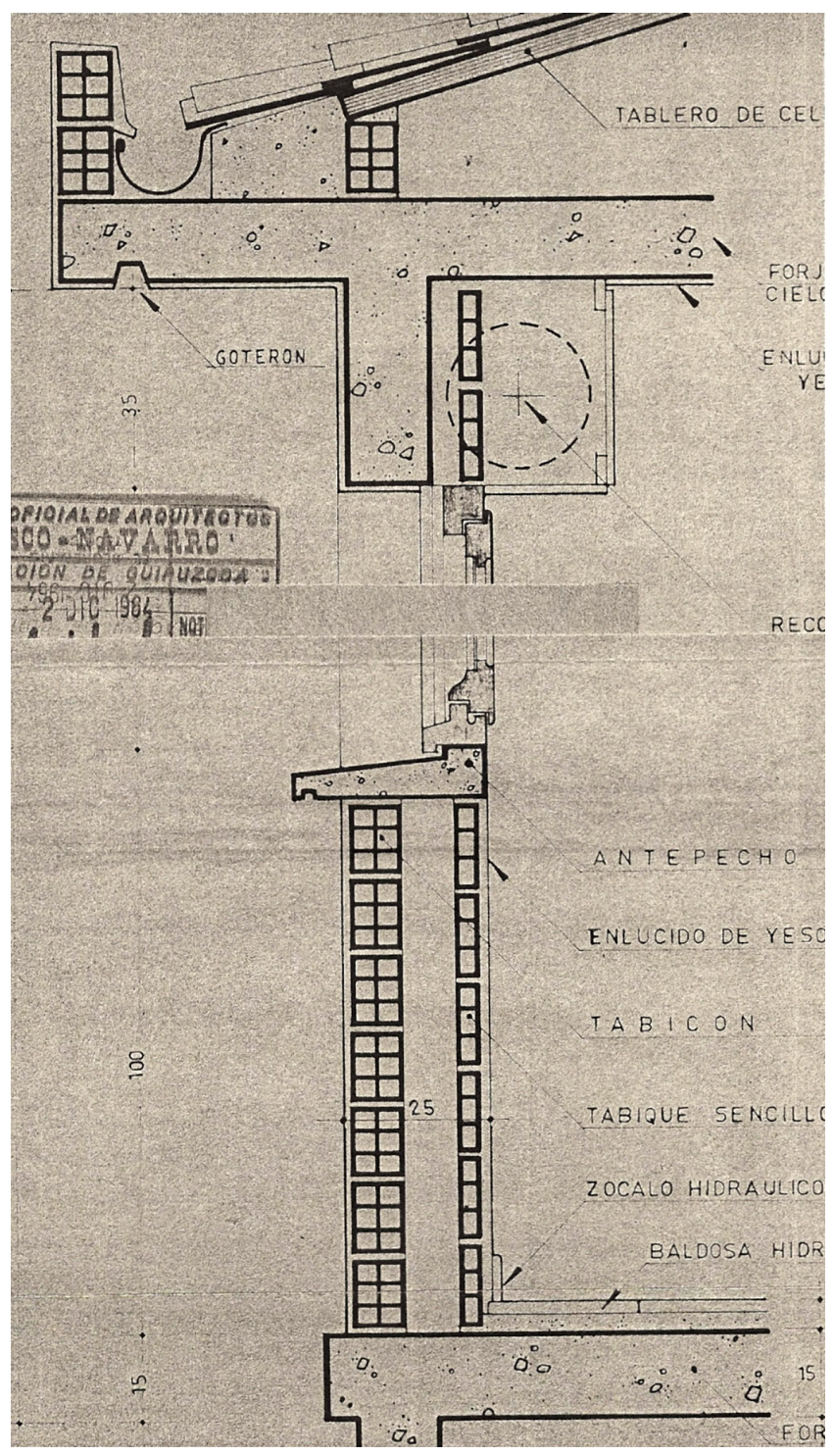

Figura 3. Detalle extraído del proyecto del conjunto residencial de 697 viviendas en Urdaburu de Errenteria, redactado en 1964 por Ramón Gabaráin.

los casos analizados) y la falta de vuelo y goterón de la última pieza de borde de baldosa. El acabado habitual del solado será la baldosa cerámica, normalmente baldosín catalán, colocada sobre una ligera capa de pendiente. En la defensa perimetral prevalecerán las barandillas metálicas formadas por una estructura tubular de hierro y las combinadas con un panelado de vidrio armado. También se pueden encontrar algunos antepechos de hormigón o fábrica de ladrillo revestidos.

\section{EL ESTADO ACTUAL DE LA FACHADA DESARROLLISTA}

La fachada convencional construida durante el periodo desarrollista ha envejecido mal y presenta, en general, importantes deficiencias. Sus múltiples errores de concepto se han visto multiplicados por una deficiente ejecución y puesta en obra. A día de hoy, muchas de ellas han llegado a una situación crítica y sus carencias y lesiones, puntuales y relativamente leves hasta hace bien poco, comienzan a acumularse y a generar situaciones realmente graves, no sólo para el propio edificio sino también para la seguridad de las personas. Sus principales problemas se resumen en los siguientes: 


\subsection{Los derivados del propio diseño}

Errores de diseño bien conocidos y que han sido suficientemente analizados en numerosas publicaciones desde finales de la década de los ochenta del siglo pasado (8) (9) (10) (11) (12) (13) (14) (15). Los más importantes son:

- La falta de libertad de movimiento: La constricción de las hojas de fachada entre los rígidos planos de forjado que limitan cada planta, condiciona y coarta los necesarios movimientos relativos entre ambos elementos constructivos.

- El insuficiente apoyo de la hoja exterior: La falta de aplomo de los frentes estructurales, un error habitual y propio de una época caracterizada por las prisas y el escaso control de la ejecución, provoca diferencias en la dimensión de la superficie de apoyo de la fábrica exterior en las diferentes plantas generando una incorrecta transmisión de las cargas (obsérvese el escaso apoyo del tabicón exterior de la Figura 2).

- El revestimiento de los frentes estructurales: Cuando la fábrica exterior queda enrasada con el frente estructural, los acabados continuos acusan el desigual comportamiento de los materiales del soporte generándose fisuras en la junta. En los frentes estructurales revestidos mediante una rasilla o una plaqueta de ladrillo la discontinuidad material queda resuelta pero surgen otros problemas como la falta de apoyo o adherencia al soporte, la recepción de cargas no previstas, las diferencias de espesor, etc. Las consecuencias se traducen en manchas superficiales, deformaciones, fisuras, aplastamientos e, incluso, desprendimientos. Las fachadas de ladrillo caravista, desprovistas de cualquier otro revestimiento adicional, son las más expuestas a sufrir este tipo de lesiones.

\subsection{La pérdida de características del revestimiento exterior}

Con el paso de las décadas, las prestaciones de los diferentes materiales de acabado han ido disminuyendo. Los enfosca- dos se han fisurado y cuarteado; los morteros de agarre se han disgregado perdiendo adherencia; las plaquetas, mosaicos vítreos y similares, incluidas las juntas, se han fisurado; la impermeabilidad de los materiales de acabado ha disminuido o desaparecido; el acabado exterior de los ladrillos caravista se ha deteriorado, etc. Todo ello ha provocado una degradación generalizada y un agotamiento de la solución original.

\subsection{El deterioro del perímetro estructural}

Las lesiones se concentran en la superficie de los elementos estructurales de hormigón y afectan, principalmente, a los pilares, vigas, forjados y losas de balcones y aleros situados en la envolvente del edificio (Figura 4).

El origen de las lesiones se encuentra en la oxidación de la armadura superficial de los elementos estructurales perimetrales y sus consecuencias pueden llegar a ser espectaculares. La presión ejercida por el incremento de volumen de la armadura dañada sobre el hormigón provoca la fisuración, el agrietamiento y el estallido final de este material y termina arrastrando, de forma irremediable, su acabado exterior (Figura 5). Se trata del problema más grave que, a día de hoy, presentan los edificios construidos durante el periodo desarrollista ya que, además de afectar a otros elementos constructivos, está poniendo en serio riesgo la seguridad de las personas por el alto riesgo de caída de cascotes a la vía pública.

La filtración progresiva de oxígeno del aire y de humedad al interior de los elementos estructurales, verdadera causante de la corrosión por oxidación de las armaduras, se produce por dos vías. Por un lado, los poros y fisuras de los revestimientos exteriores permiten la filtración directa de agua de lluvia. Algo especialmente significativo en el caso de los vuelos de balcón donde la sistemática falta de impermeabilización bajo un solado deteriorado por el paso del tiempo facilita

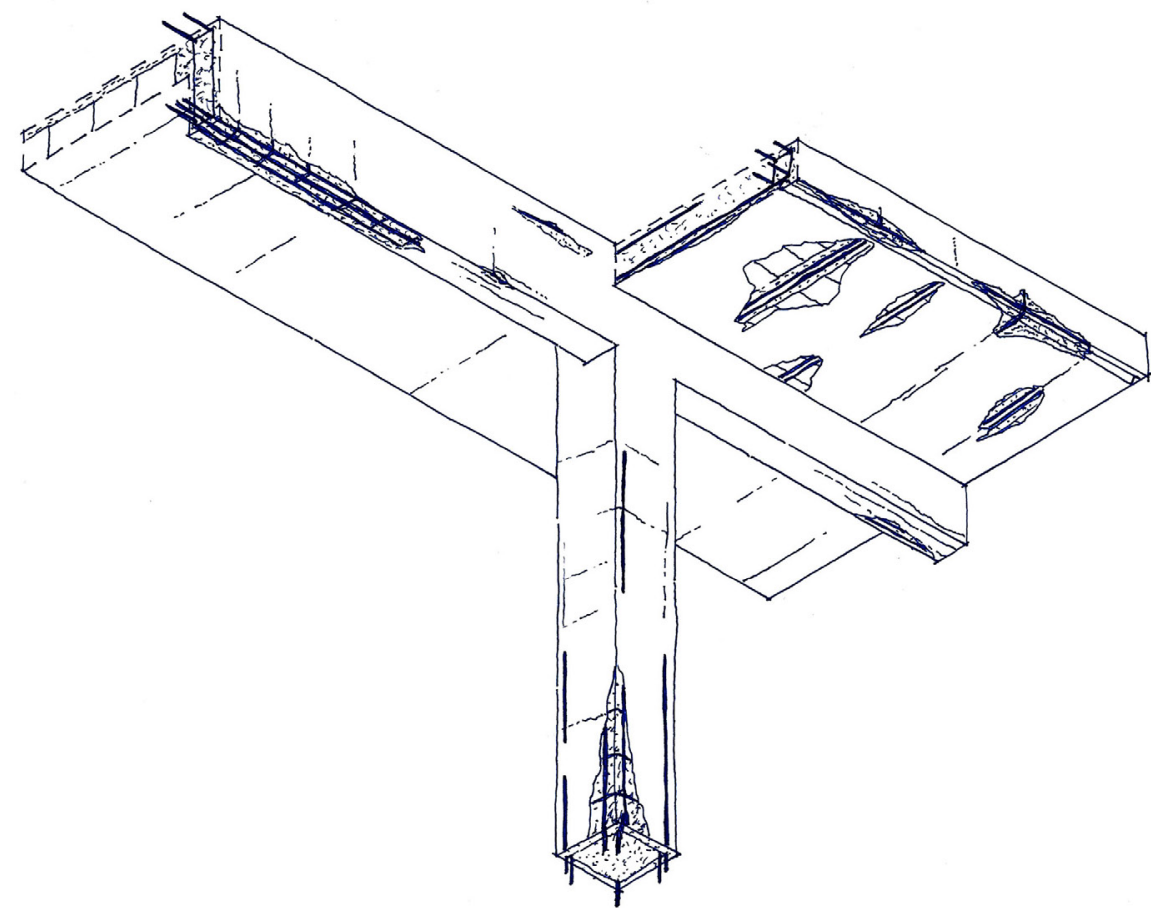

Figura 4. Representación gráfica de los efectos producidos por la oxidación de las armaduras en los elementos estructurales situados en la envolvente de fachada. 


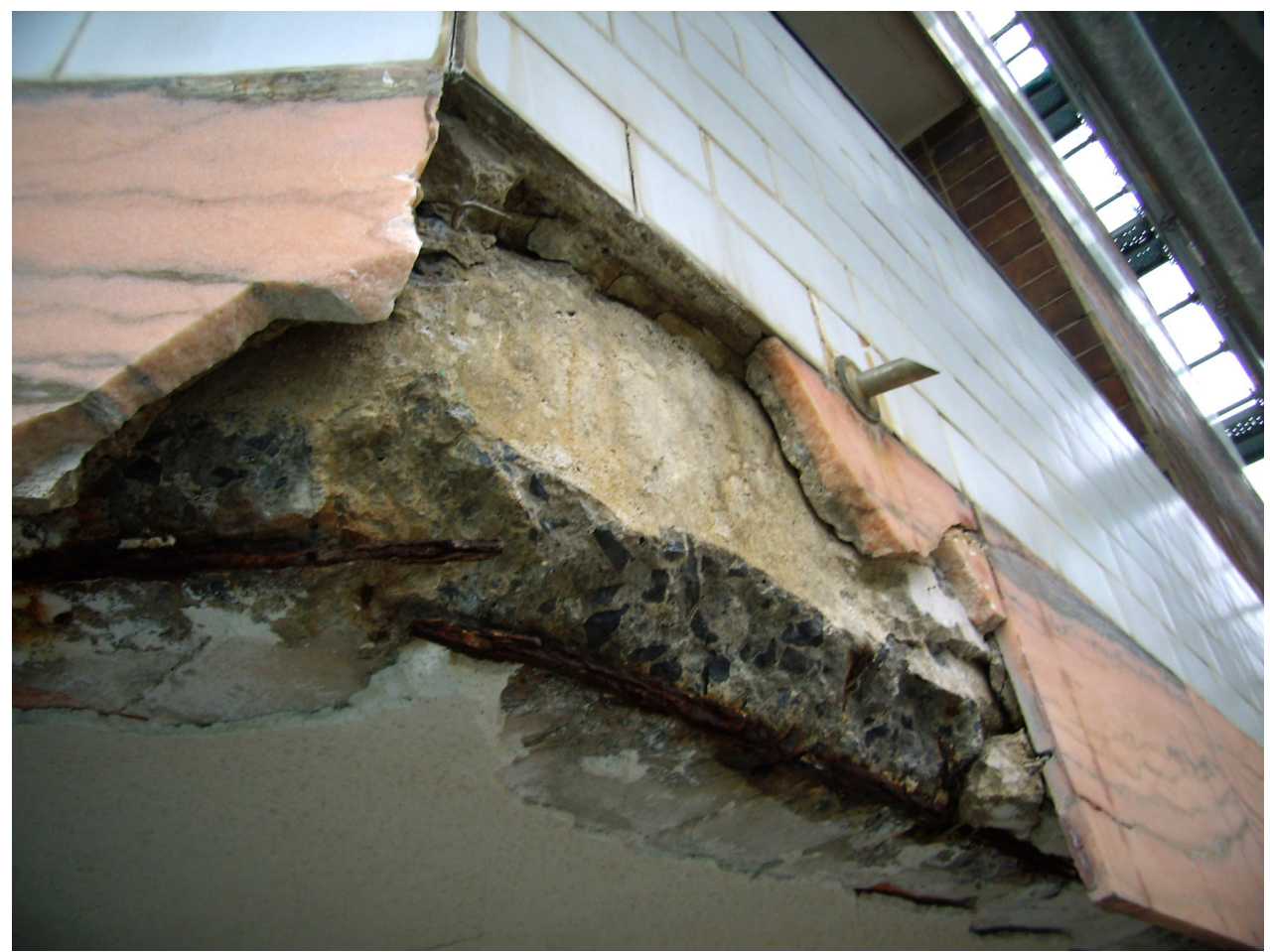

Figura 5. Rotura y caída de parte del aplacado de piedra y del hormigón de la viga de borde del forjado de uno de los balcones del edificio de 20 viviendas en Zinkunegi, 7 de Zarautz.

la humectación del soporte estructural. La otra causa fundamental que favorecerá la desprotección de la armadura ante la agresión ambiental será la carbonatación del hormigón, una reacción química natural e inevitable que se produce cuando el $\mathrm{CO}_{2}$ del aire penetra a través de la estructura porosa del hormigón y carbonata el hidróxido de calcio $\mathrm{Ca}(\mathrm{OH})_{2}$ del cemento generando carbonato cálcico más agua $\left(\mathrm{CaCO}_{3}\right.$ $+\mathrm{H}_{2} \mathrm{O}$ ). Dicha reacción provoca una disminución del $\mathrm{pH}$ del hormigón, neutralizándolo. Como si estuvieran directamente expuestas al exterior, las barras de armado comienzan a sufrir un proceso de oxidación progresivo e irreversible. El grado de afectación dependerá, fundamentalmente, de tres factores:

- El grado de superficialidad del armado perimetral: El escaso espesor del hormigón de recubrimiento se deberá, en casi todos los casos, a un simple pero muy habitual error de ejecución durante aquellos años: la deficiente e irregular colocación de las barras de armado, prácticamente adosadas al encofrado, sin respetar unas mínimas dimensiones de separación y en ausencia de elementos separadores.

- La calidad del hormigón: La calidad de los hormigones fabricados por aquellos años fue muy desigual. Mientras algunos han llegado hasta nuestros días en perfecto estado, otros están pagando un alto precio por dosificaciones mal compensadas, el uso de áridos excesivamente gruesos, el empleo de arenas mal lavadas, la ejecución de mezclas poco homogéneas y con un alto grado de disgregación, un incorrecto vertido y proceso de compactado, etc.

- El ambiente exterior: La localización del edificio resulta también determinante. En las inspecciones realizadas se ha observado que los edificios situados en municipios costeros, con un ambiente del tipo niebla marina o similar (16), presentan un peor estado. Incluso en estas localidades, la mayor o menor proximidad al frente marítimo influye significativamente en el grado de afectación.
La corrosión de la armadura debido al ion cloruro, cuyos efectos pueden llegar a ser aún más destructivos, tendrá mucha menor incidencia. El porcentaje de cloruros obtenido en los ensayos de laboratorio realizados sobre varias muestras extraídas del perímetro estructural de dos de los edificios más dañados de la localidad costera de Zarautz, la torre de Vista Alegre de Juan Manuel Encío y Luis Peña Ganchegui de 1958 (17) y el conjunto residencial Ondar Zelai de Roberto Martínez Anido de 1970 (18), se sitúa dentro de los valores considerados aceptables según la actual EHE. No así los de la profundidad de carbonatación, que dan valores muy altos en todas y cada una de las muestras extraídas de ambos edificios.

\subsection{El deterioro de los huecos y vuelos de fachada}

Las fisuras, grietas y desprendimientos que se observan en los alféizares y dinteles, tanto prefabricados como realizados in situ, obedecen a la misma causa: la oxidación de la armadura interior. En cuanto a los vuelos, además de las lesiones estructurales, hay que destacar la pérdida de impermeabilidad de la cara superior de los balcones, confiada únicamente al solado; la corrosión sufrida por las barandillas de hierro, acentuada en los apoyos de las montantes que se insertan directamente en el soporte; y la ausencia generalizada de albardillas en el caso de los antepechos de balcón.

\subsection{La falta de aislamiento}

Además de las incalculables pérdidas energéticas generadas a lo largo del tiempo debido a su endeblez térmica, están surgiendo una serie de nuevos problemas. Es el caso de las condensaciones, prácticamente inexistentes hasta hace bien poco y que se han multiplicado desde el momento en que las viviendas han comenzado a ser reformadas. Los hasta entonces inexistentes puentes térmicos se potencian y si, como ocurre en ocasiones, las obras de reforma se realizan en 
ausencia de un control técnico e incluyen la introducción descontrolada de un material aislante en el interior de la cámara, el riesgo aumenta de forma exponencial.

\section{RESUMEN DE DATOS Y SÍNTESIS DE RESULTADOS}

En la Tabla 1 se resumen los principales materiales, sistemas y elementos constructivos empleados en la construcción de los 128 conjuntos residenciales analizados, su estado de conservación y el grado de intervención sufrido a lo largo del tiempo.

En los gráficos A, B y C (Figura 6) se realiza un análisis porcentual de los principales datos de la Tabla 1.

El gráfico A refleja la conformación original y el estado de conservación de tres de los principales elementos constructivos de la fachada: la hoja exterior, el perímetro del hueco y la estructura de los balcones. Sin considerar las deficiencias

Tabla 1. Resumen.

\begin{tabular}{|c|c|c|c|c|c|c|c|c|c|c|c|c|c|}
\hline \multirow{2}{*}{\multicolumn{6}{|c|}{ Total conjuntos residenciales analizados (1) : [128] }} & \multicolumn{5}{|c|}{ Estado de conservación (2) } & \multicolumn{3}{|c|}{$\begin{array}{l}\text { Reparaciones y/o } \\
\text { reformas realizadas }\end{array}$} \\
\hline & & & & & & $\mathbf{I}$ & II & III & IV & $?$ & $\underset{\text { Sin }}{\text { reformar }}$ & Parcial & Completa \\
\hline \multirow{8}{*}{$\begin{array}{l}\text { Hoja } \\
\text { exterior }\end{array}$} & \multirow{6}{*}{\multicolumn{2}{|c|}{$\begin{array}{l}\text { Fábrica de } \\
\text { Ladrillo } \\
\text { Hueco Doble } \\
\text { revestido [96] }\end{array}$}} & \multicolumn{2}{|c|}{$\begin{array}{l}\text { Revestimientos } \\
\text { continuos [42] }\end{array}$} & $\begin{array}{l}\text { Pintura, Tirolesa, } \\
\text { Granulite [42] }\end{array}$ & 9 & 3 & 25 & 2 & 3 & 8 & 21 & 13 \\
\hline & & & \multirow{5}{*}{\multicolumn{2}{|c|}{$\begin{array}{l}\text { Aplacados (como material } \\
\text { predominante en el } \\
\text { conjunto) }[54]\end{array}$}} & Plaqueta gres [6] & 4 & 1 & 1 & - & - & 2 & 1 & 3 \\
\hline & & & & & Mosaico vítreo [23] & 4 & 2 & 12 & 4 & 1 & 10 & 4 & 9 \\
\hline & & & & & Ladrilleta [12] & 5 & 1 & 6 & - & - & 8 & 3 & 1 \\
\hline & & & & & Piedra natural [7] & 3 & - & 2 & 2 & - & 4 & 2 & 1 \\
\hline & & & & & Baldosa hidráulica [6] & - & 1 & 4 & - & 1 & 1 & 4 & 1 \\
\hline & \multirow{2}{*}{\multicolumn{2}{|c|}{\begin{tabular}{|l|} 
Fábrica de \\
Ladrillo \\
Caravista [32]
\end{tabular}}} & \multicolumn{3}{|c|}{ Ladrillo Caravista [22] } & 9 & 2 & 10 & 1 & - & 12 & 6 & 4 \\
\hline & & & \multicolumn{3}{|c|}{$\begin{array}{l}\text { Combinada: ladrillo caravista + otros } \\
\text { materiales [10] }\end{array}$} & 4 & 3 & 1 & - & 2 & 5 & 2 & 3 \\
\hline \multirow{2}{*}{\multicolumn{4}{|c|}{ Cámara de aire }} & \multicolumn{2}{|c|}{ Sin aislamiento [126] } & 4 & - & - & - & 122 & 126 & - & - \\
\hline & & & & \multicolumn{2}{|c|}{ Con aislamiento [2] } & - & - & - & - & 2 & 2 & - & - \\
\hline \multirow{2}{*}{\multicolumn{4}{|c|}{ Hoja interior }} & \multicolumn{2}{|c|}{ Ladrillo Hueco Sencillo [110] } & - & - & - & - & 110 & 110 & - & - \\
\hline & & & & \multicolumn{2}{|c|}{ Ladrillo Hueco Doble [18] } & - & - & - & - & 18 & 18 & - & - \\
\hline \multirow{13}{*}{$\begin{array}{l}\text { El hueco } \\
\text { de fachada }\end{array}$} & \multirow{6}{*}{ Dintel } & \multicolumn{2}{|c|}{ Visto [29] } & \multicolumn{2}{|c|}{ Viga de canto [29] } & 5 & 6 & 13 & - & 5 & 16 & 9 & 4 \\
\hline & & & & \multicolumn{2}{|c|}{ Viga de canto [66] } & 6 & 6 & 21 & - & 33 & 28 & 24 & 14 \\
\hline & & & & \multicolumn{2}{|c|}{$\begin{array}{l}\text { Elemento prefabricado cerámico } \\
\text { o de hormigón [24] }\end{array}$} & 4 & 1 & 4 & - & 15 & 11 & 10 & 3 \\
\hline & & {$[99$} & ] & Perfil de acero & & - & - & - & - & 1 & 1 & - & - \\
\hline & & & & Ladrillo carav. & dintel in situ [7] & 1 & 2 & 4 & - & - & 4 & 1 & 2 \\
\hline & & & & Arco de correa & e LHD [1] & - & 1 & - & - & - & - & 1 & - \\
\hline & & & & Piedra artificia & hormigón [91] & 10 & 24 & 51 & 5 & 1 & 34 & 49 & 8 \\
\hline & Alféizar & Vist & to [97] & Ladrillo caravi & a a sardinel [6] & - & 1 & 5 & - & - & 6 & - & - \\
\hline & & $\operatorname{Rev}$ & restido [31] & Soporte cerám & $0+$ aplacado [31] & 5 & 6 & 16 & - & 4 & 9 & 10 & 12 \\
\hline & & & pintería & Madera [104] & & 1 & 19 & - & - & 84 & - & 104 & - \\
\hline & & (3) & 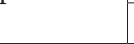 & Metálica (alum & iio) $[5]$ & 1 & 1 & - & - & 3 & - & 5 & - \\
\hline & Ventana & & & Sencillo [56] & & - & - & - & - & 56 & - & 56 & - \\
\hline & & Vid & rio (3) & Doble [29] & & - & - & - & - & 29 & - & 29 & - \\
\hline & Fstru & & & Forjado unidir & cional [121] & 23 & 15 & 53 & 5 & 25 & 50 & 51 & 20 \\
\hline & Estructura & & & Losa armada [ & & 1 & 1 & 1 & 1 & 1 & 3 & 1 & 1 \\
\hline & & & & Baldosín catalá & [79] & - & 4 & 8 & 1 & 66 & 19 & 58 & 2 \\
\hline & & Sole & ados (3) & Otros material & {$[16]$} & - & - & 3 & 2 & 11 & 1 & 16 & - \\
\hline & Acabados & & & Pintura sobre $\epsilon$ & foscado [100] & 32 & 19 & 46 & 3 & - & 27 & 47 & 26 \\
\hline $\begin{array}{l}\text { Balcones } \\
\text { (4) }\end{array}$ & & Тес & hos & Falso techo de & adera [26] & 9 & 7 & 10 & - & - & 10 & 11 & 5 \\
\hline & & & & Herrería [61] & & 7 & 39 & 15 & - & - & 4 & 53 & 4 \\
\hline & & $\begin{array}{l}\text { Bar } \\
\text { Oo7 }\end{array}$ & andillas & Herrería + vidı & armado [35] & 2 & 30 & 3 & - & - & - & 33 & 2 \\
\hline & Defensas & & & Madera [1] & & - & - & 1 & - & - & 1 & - & - \\
\hline & & $\begin{array}{l}\text { Ant } \\
{[29}\end{array}$ & epecho & Fábrica de ladr & lo + herrería [29] & & & & & & & & \\
\hline & & & & Cerámica [16] & & 4 & 8 & 4 & - & - & 8 & 5 & 3 \\
\hline $\begin{array}{l}\text { Otros } \\
\text { elementos }\end{array}$ & Celosías en & fach & ada (5) & Hormigón [22] & & 6 & 8 & 8 & - & - & 9 & 8 & 5 \\
\hline & & & & Otros material & [12] & 2 & 2 & 8 & - & - & 5 & 5 & 2 \\
\hline
\end{tabular}

(1) 128 conjuntos residenciales que incluyen 484 bloques o edificios y 13.433 viviendas.

(2) Estado de conservación: I: Aparentemente bueno. II: Deficiencias puntuales. III: Deterioro constructivo. IV: Deterioro extremo. ?: Sin poder determinar

(3) La suma de los diferentes tipos es inferior al total porque en el resto de casos no se ha podido determinar su naturaleza.

(4) Dos de los conjuntos residenciales analizados no cuentan con balcones.

(5) Inexistentes en algunos de los conjuntos residenciales analizados. En algunos casos, no se ha podido determinar su existencia. 
puntuales, cabe destacar que más de la mitad de las fachadas $(54,7 \%)$ presentan un deterioro constructivo generalizado o extremo de su acabado exterior. En cuanto al hueco de fachada, más de un $60 \%$ de los alféizares están dañados así como un tercio de los dinteles, algo realmente preocupante teniendo en cuenta que en el 74,2 \% de los casos éstos están formados por las vigas de canto perimetrales. En cuanto a la estructura de los balcones, casi la mitad (48 \%) presentan fisuras, grietas o desprendimientos en su base o frente estructural.

El gráfico B resume el grado de utilización de otros elementos y materiales en la construcción original de los edificios analizados. No se valora su estado actual ya que muchos de ellos han ido siendo sustituidos y otros no son accesibles visualmente.

Por último, del gráfico $\mathrm{C}$ se deduce que algo más de la mitad de los edificios analizados han sufrido diferentes procesos de reforma, en su mayor parte parciales o puntuales. Los elementos constructivos que han sido completamente reparados y/o reformados representan alrededor de una quinta parte. La rehabilitación integral del conjunto de la envolvente verti- cal es el gran reto pendiente de la inmensa mayoría de estos edificios.

\section{CONCLUSIONES}

Al ser cuestionados sobre la calidad constructiva de los edificios del periodo analizado, la opinión de todos los personajes entrevistados durante el proceso de investigación es coincidente: en un contexto y unas circunstancias muy determinadas y utilizando lo que en aquella época podía considerarse tecnología punta, se construyó como mejor se sabía y podía. El edificio desarrollista respondía adecuadamente a los requisitos de calidad y confort exigidos por aquellos años y tanto las soluciones constructivas como los materiales empleados se adecuaban perfectamente a los nuevos tiempos. Ahora bien, pasadas cuatro o cinco décadas, resulta igual de evidente que estos edificios han envejecido mal y que no responden a las exigencias y necesidades de la sociedad actual.

La fachada es el ejemplo más claro de este deterioro progresivo. Una solución de fachada que ya comenzó a ser firmemente cuestionada en los últimos años del siglo pasado por

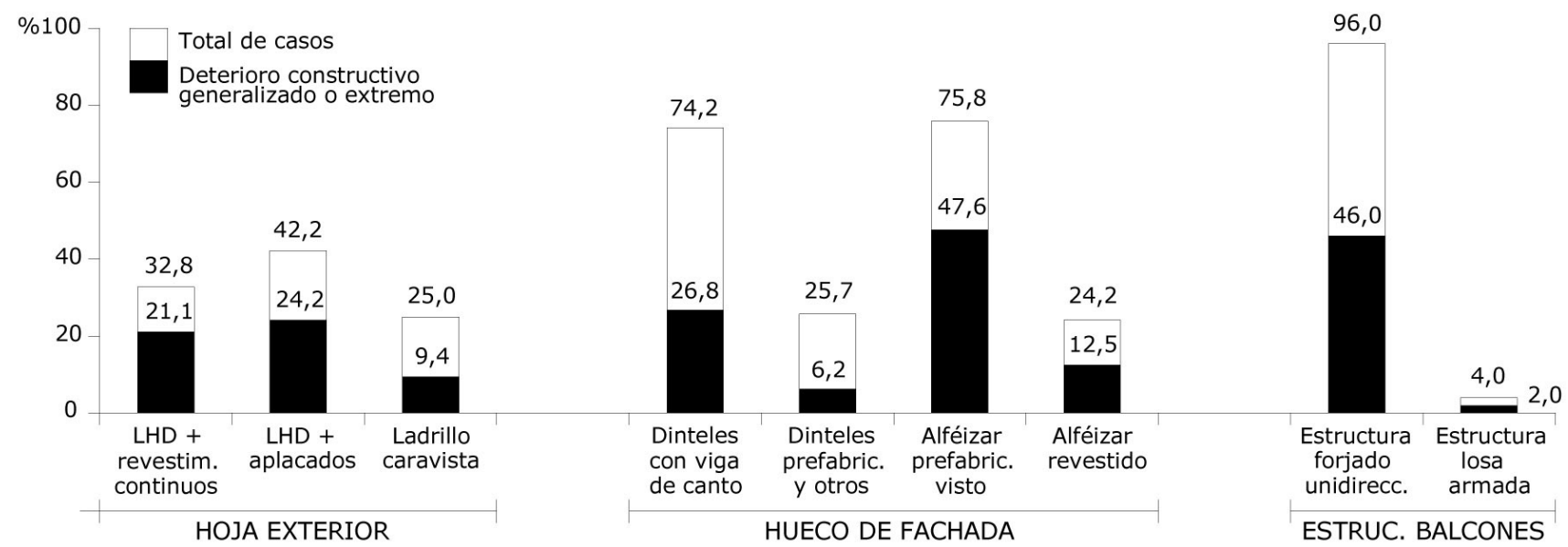

A. ESTADO DE CONSERVACION (\%)

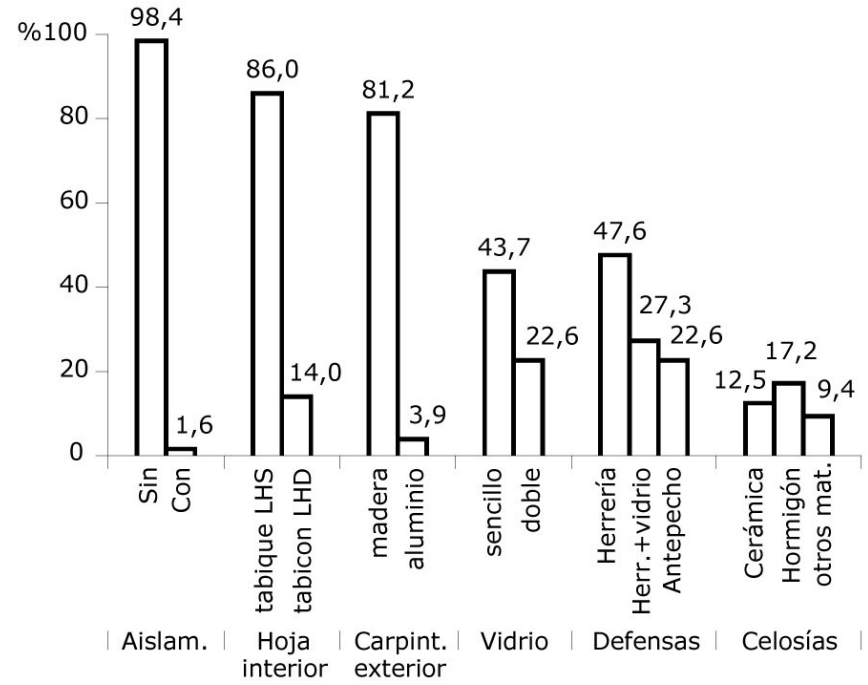

B. EMPLEO DE OTROS ELEMENTOS (\%)

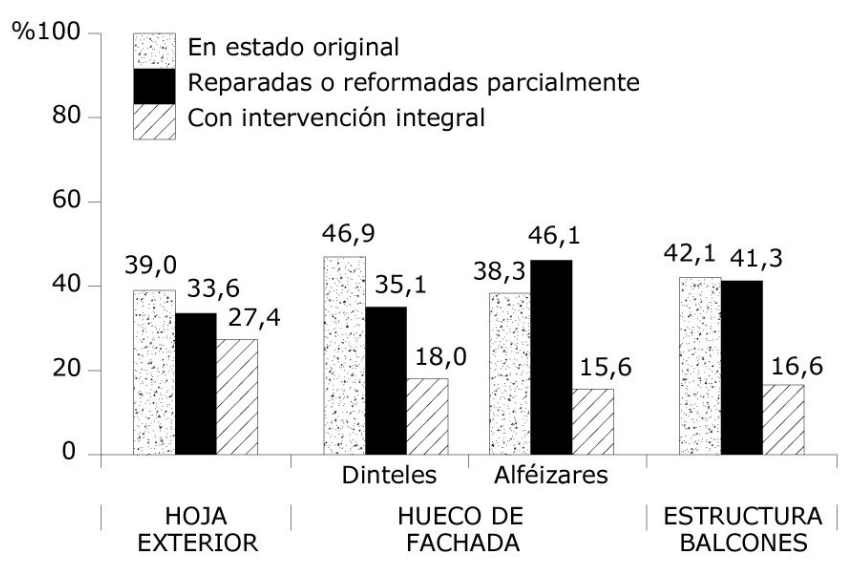

C. GRADO DE INTERVENCION (\%)

Figura 6. Síntesis de resultados. 
diferentes autores. Sirva como ejemplo esta contundente frase de J. Monjo de 1990 refiriéndose a la solución convencional: «...totalmente rechazable la solución normalmente utilizada que, por ello, se convierte en un "error sistemático" de diseño constructivo sin aparente alternativa, refrendada, sin embargo, por multitud de publicaciones e, incluso, de normativa» (8). No se entiende, por lo tanto, que pasadas varias décadas continúe siendo la solución más utilizada en la construcción actual y que normativas recientes como el Código Técnico de la Edificación (CTE) sigan apoyándose en ella (sirvan como ejemplo las Figuras 2.8, 2.9 y 2.10 del DB HS-1) pretendiendo resolver y dar respuesta a errores de concepto, de por sí, irresolubles. Una fachada que muestra claros síntomas de un agotamiento constructivo generalizado y cuyo ciclo de vida debería de darse por culminado siendo desterrada definitivamente, como solución, de la construcción actual y futura.

Pero a los graves errores de diseño y a las lesiones, algunas de las cuales, conviene insistir, están poniendo en riesgo la seguridad de las personas, se suman sus carencias. La más importante, la falta de aislamiento. Las exigencias actuales en materia de sostenibilidad y eficiencia energética hacen que este hecho, absolutamente irrelevante en el momento de su construcción, adquiera a día de hoy una importancia enorme.

Todo ello va a hacer que la forma de plantear los procesos de rehabilitación de este tipo de edificios cambie, en adelante, de forma radical. Si hasta ahora la mayoría de las intervenciones se limitaban a la reparación de los elementos dañados o a una simple sustitución de los acabados de fachada, las nuevas exigencias normativas y de confort van a hacer que la rehabilitación integral de la envolvente se imponga y que la reducción de la demanda energética se convierta en un objetivo ineludible.

En cuanto a las posibles actuaciones rehabilitadoras, dada la dificultosa gestión de cualquier planteamiento de derribo y posterior reposición de la hoja exterior de muchas de ellas, única solución definitiva en el caso de las fabricas vistas que deberían ser reconstruidas mediante hojas pasantes armadas, dos son los sistemas que, a día de hoy, parecen imponerse: los revestimientos continuos adheridos tipo SATE (Sistemas de Aislamiento Térmico por el Exterior) y las fachadas ventiladas en todas sus variantes. Dejando de lado las evidentes mejoras y prestaciones tecnológicas que cualquiera de ellas pueden llegar a ofrecer, ante la singular característica que ambas comparten, la de ocultar completamente el acabado original, es necesario plantear una serie de cuestiones que habitualmente no se introducen en el debate:

¿Se adaptan bien, desde el punto de vista compositivo y constructivo, a las diferentes soluciones de fachada del periodo desarrollista?

Al tratarse de nuevas pieles especialmente diseñadas para la resolución de paños frontales, lisos y continuos, ¿qué ocurre con los puntos singulares y, más concretamente, con el recerco de las ventanas, auténtico punto crítico debido a sus diferencias dimensionales y la necesidad de variar el espesor y tipo de aislamiento?

¿Sirven para solucionar las lesiones existentes o se limitan a ocultarlas? Desde luego, la superposición de una nueva piel, con su intrínseca y sugerente cualidad de ocultar lo preexistente, puede generar cierta relajación a la hora de afrontar su costosa y compleja reparación.

¿A quién corresponde la decisión de optar por la mejor solución? ¿A los técnicos, a la Administración, a las Comunidades de Propietarios...?

Y una última y fundamental, ¿Cómo va a afectar a la memoria arquitectónica y constructiva de nuestros pueblos y ciudades la constante y sistemática repetición de nuevas soluciones totalmente estandarizadas, moduladas e industrializadas?

Preguntas que no tienen fácil respuesta y que requieren de una reflexión conjunta por parte de todos los agentes involucrados. Y para ayudar a dar respuesta a estas y otras cuestiones que, sin duda, irán surgiendo en los próximos tiempos, se antoja necesario realizar, al igual que se hace con el patrimonio de épocas anteriores, una tipificación y catalogación de la multitud de edificios construidos durante aquel denostado periodo que resulte útil a la hora de establecer criterios de actuación en futuros procesos de rehabilitación.

La ley es muy clara al trasladar a los propietarios de las viviendas la responsabilidad última de los posibles daños que la falta de mantenimiento del edificio pueda ocasionar tanto al propio edificio como a sus usuarios y terceras personas. Por ello, la rehabilitación de estos edificios no debería posponerse en el tiempo y tendría que ser afrontada de forma urgente. Una circunstancia que, en un contexto de crisis económica de final incierto, puede convertirse en un auténtico problema social ya que muchas Comunidades de Vecinos se están viendo y se van a ver muy limitadas económicamente a la hora de afrontar no ya una rehabilitación integral de coste elevado sino cualquier mínima y urgente reparación.

\section{AGRADECIMIENTOS}

El artículo sintetiza la parte dedicada a la fachada en la Tesis Doctoral (19) que analiza el proceso constructivo y el estado actual de los edificios de vivienda colectiva construidos durante el periodo desarrollista en el Territorio Histórico de Gipuzkoa. El autor agradece la ayuda y las sugerencias recibidas por parte de los profesores D. Joseba Escribano y D. Alberto Zulueta (Universidad del País Vasco), D. ${ }^{a}$ Carmen Rodríguez (Universidad de Sevilla), D. ${ }^{\text {a }}$ Mercedes del Río (Universidad Politécnica de Madrid) y D. Cesar Díaz y D. Josep Ignasi de Llorens (Universitat Politècnica de Catalunya). Un recuerdo y agradecimiento muy especial a los diecisiete personajes entrevistados para el presente trabajo: los arquitectos D. Juan M. ${ }^{a}$ Aguirre, D. Ander Basterretxea, D. Francisco Bernabé, D. Juan Manuel Encío, D. José Ramón Marticorena, D. Xabier Peñalba, D. José Luis Pla, D. Armando Roca, D. Jesús M. ${ }^{a}$ Tanco y D. Xabier Unzurrunzaga; el aparejador D. José UribeEtxeberria; los promotores, contratistas y fabricantes de materiales D. José Luis Ayestarán (Inmobiliaria Orio), D. Pedro Eizaguirre y D. Alberto Eizaguirre (Construcciones Eizaguirre y Prefabricados Navarros S.A.), D. Tomás Liceaga (Hormigones Euskalduna) y D. Andrés Tellería (Tejas y Ladrillos del Oria S.A.); y D. Gervasio Juaristi, Concejal y Alcalde de Zarautz entre 1963 y 1976. 


\section{REFERENCIAS}

(1) Auñamendi. (1984). Enciclopedia General Ilustrada del País Vasco. (1984). Vol. XVII, pp. 324-25. Donostia: Ed. Auñamendi.

(2) Gobierno Vasco. (2010). Plan estratégico de la CAPV de rehabilitación de edificios y regeneración urbana 201O-2013, (pp. 24-29). Dpto. de Vivienda, Obras Públicas y Transportes.

(3) Ministerio de la vivienda. (1960). Pliego de Condiciones Técnicas de la Dirección General de Arquitectura 196o, (pp. 89-92). Madrid: Ministerio de la vivienda - Dirección General de Arquitectura y Tecnología de la Edificación.

(4) Entrevista realizada al arquitecto Armando Roca García (1934, Lleida) en su estudio de Tolosa (Gipuzkoa) el 19 de octubre de 2010.

(5) Ros, J.M. (2005). La fábrica de doble hoja en Madrid, un siglo de cerramiento moderno. Informes de la Construcción, 56(495): 57-71, doi: http://dx.doi.org/10.3989/ic.2005.v57.i495.455.

(6) Presidencia del Gobierno. (1975). Decreto 1490/1975, de 12 de junio, por el que se establecen medidas a adoptar en las edificaciones con objeto de reducir el consumo de energía. Boletín Oficial del Estado, $\mathrm{n}^{0}$ 165. España.

(7) Ministerio de la Vivienda. (1965). Seminarios del INV. 1. Viviendas (pp. 53-54). Madrid: Ediciones del INV.

(8) Monjo, J. (1990). Errores sistemáticos en el diseño constructivo de fachadas. Informes de la Construcción, 42(407): 5372, doi: http://dx.doi.org/10.3989/ic.1990.v42.i407.1449.

(9) Adell, J.M. (2000). Arquitectura sin fisuras. pp. 6-7. Madrid: Editorial Munilla-Lería.

(10) Adell, J.M. (2000). Arquitectura sin fisuras. pp. 26-61. Madrid: Editorial Munilla-Lería.

(11) Díaz, C. (2006). Patología e intervención en fachadas de ladrillo visto. En Actas del $2^{\circ}$ Encontro sobre patologia e reabilitação de edifícios PATORREB 2006. Porto.

(12) Monjo, J. (2010). Patología de cerramientos y acabados arquitectónicos. Cap. IV, pp. 196-199. Madrid: Editorial Munilla-Lería.

(13) Monjo, J. (2010). Patología de cerramientos y acabados arquitectónicos. Cap. IV, pp. 209-210. Madrid: Editorial MunillaLería.

(14) Monjo, J. (2010). Patología de cerramientos y acabados arquitectónicos. Cap. IV, pp. 228. Madrid: Editorial MunillaLería.

(15) Monjo, J. (2010). Patología de cerramientos y acabados arquitectónicos. Cap. IV, pp. 244-246. Madrid: Editorial Munilla-Lería.

(16) Talero, R. (1998).Tratado de rehabilitación. Tomo 3, Patología y técnicas de intervención. Elementos estructurales. Cap. IV, pp. 332-334. Madrid: Editorial Munilla-Lería.

(17) GIKESA. (2005). Expediente 2005-0407. Acta $n^{\circ} 78008$. Donostia.

(18) LABEIN TECNALIA. (2002). Documento $n^{\circ}$ B118/o2.o8-IN-CM-O1 (vo2). Derio.

(19) Lizundia, I. (2012). La construcción de la arquitectura residencial en Gipuzkoa durante la época del Desarrollismo (Tesis Doctoral). Universidad del País Vasco EHU/UPV. 